Stavropoulos, V., Griffiths, M.D., Burleigh, T.L., Kuss, D.J., Doh, Y.W. \& Gomez, R. (2018). Flow on the Internet: A longitudinal study of internet addiction symptoms in adolescence. Behavior and Information Technology, https://doi.org/10.1080/0144929X.2018.1424937

\title{
Flow on the Internet: A Longitudinal Study of Internet Addiction Symptoms During
}

\section{Adolescence}

\author{
Vasileios Stavropoulos ${ }^{1,2}$, Mark D. Griffiths ${ }^{3}$, Tyrone L. Burleigh ${ }^{2}$, Daria J. Kuss ${ }^{3}$, Young \\ Yim Doh ${ }^{4}, \&$ Rapson Gomez ${ }^{2}$ \\ ${ }^{1}$ University of Athens, Greece, ${ }^{2}$ Federation University, Australia, ${ }^{3}$ Nottingham Trent \\ University, UK, ${ }^{4}$ Yonsei University, Korea.
}

Vasileios Stavropoulos, Federation University Australia, PO Box 663, Ballarat, VIC, 3353 Australia. v.stavropoulos@federation.edu.au.+61(03) 53276273

Mark D. Griffiths, Nottingham Trent University, 50 Shakespeare Street, Nottingham, NG1 4FQ. mark.griffiths@ntu.ac.uk.+44(0)115848 2401.

Corresponding Author: Tyrone L. Burleigh, 612 Havelock Street, Blackhill, Victoria 3350. tyrone.1.burleigh@gmail.com.+61412218117

Daria Kuss, Nottingham Trent University, 50 Shakespeare Street, Nottingham, NG1 4FQ. daria.kuss@ntu.ac.uk.+44(0)115848 4153

Young Yim Doh, KAIST GSCT, 291, Daehak-ro, Yuseong-gu, Daejeon 34141 Republic of Korea. yydoh@kaist.ac.kr.+82-42-350-2994

Rapson Gomez, Federation University Australia, PO Box 663, Ballarat, VIC, 3353 Australia. rapson.gomez@federation.edu.au.+61(03) 53276087

Compliance with Ethical Standards 


\section{Funding:}

Data collection in Greece for this study has been co-financed by the European Union (European Social Fund - ESF) and Greek national funds, under the Operational Program "Education and Lifelong Learning" of the National Strategic Reference Framework (NSRF) Research Funding Program: Heracleitus II

\section{Authors' Contribution:}

VS contributed to the data collection and analyses

RG contributed to the literature review and hypotheses formulation

MG contributed to the structure, sequencing of theoretical arguments, and paper editing

TB contributed to the data analyses

DK contributed to the literature review

YD contributed to the literature review

\section{Conflict of Interest:}

The authors of the present study do not report any conflict of interest.

Ethical Standards - Animal Rights:

All procedures performed in the study involving human participants were in accordance with the ethical standards of the institutional and/or national research committee and with the 1964 Helsinki declaration and its later amendments or comparable ethical standards. This article does not contain any studies with animals performed by any of the authors.

\section{Informed consent:}

Informed consent was obtained from all the parents and the guardians of the adolescents participating in the study, as well as the individual participants themselves.

\section{Confirmation Statement:}

Authors confirm that this paper has not been either previously published or submitted simultaneously for publication elsewhere.

Copyright:

Authors assign copyright or license the publication rights in the present article 
Flow on the Internet: A Longitudinal Study of Internet Addiction Symptoms During Adolescence 


\begin{abstract}
Internet Addiction (IA) constitutes an excessive internet use behavior with significant impact on the user's wellbeing. Online flow describes the users' level of being absorbed by their online activity. The present study investigated age-related, gender, and flow effects on IA in adolescence. The sample comprised 648 adolescents who were assessed twice at age 16 and 18 years. IA was assessed using the Internet Addiction Test and online flow was assessed using the Online Flow Questionnaire. A three-level hierarchical model estimated age-related, gender, and online flow effects on IA symptoms and controlled for clustered random effects. IA symptoms decreased over time (for both genders) with a slower rate in males. Online flow was associated with IA symptoms and this remained consistent over time. Findings expand upon the available literature suggesting that IA symptoms could function as a developmentrelated manifestation at the age of 16 years, while IA-related gender differences gradually increase between 16 and 18 years. Finally, the association between online flow and IA symptoms remained stable independent of age-related effects. The study highlights individual differences and provides directions for more targeted prevention and intervention initiatives for IA.
\end{abstract}

Keywords: Internet addiction, Online addictions, Adolescent addiction, Longitudinal study, Flow online

\title{
1. Introduction
}


The Internet has become an essential tool in the social lives of adolescents in many countries around the world (Stavropoulos, Gentile \& Motti-Stefanidi, 2016b). However, the abuse of the medium constitutes an ongoing risk for a minority of adolescents in terms of achievement of developmental tasks and general adaptation (Anderson, Steen, \& Stavropoulos, 2016). Furthermore, there appears to be a continuum between adaptive and maladaptive Internet use which needs to be carefully considered (Stavropoulos, Alexandraki \& Motti-Stefanidi, 2013a). Various terms have been suggested to depict the contradiction/continuum between the impact of Internet deprivation on psychological wellbeing and the deprivation of wellbeing because of excessive Internet use, respectively (Kuss, Griffiths, Karila, \& Billieux, 2014).

The digital divide describes the inequality between groups in terms of access to, and use or knowledge of information and communication technologies (Sui, Goodchild, \& Elwood, 2013). IA has been described as a behavior that impacts the user's ability to control online use to the extent that it can cause relational, occupational, and social problems (Kuss et al., 2014). In an attempt to expand upon the available literature in regards to factors that increase the severity of IA symptoms in adolescence, the present study explored three areas that, to the best of the authors' knowledge, have never been previously investigated and/or require further empirical study. These refer to age-related changes, the development of gender differences over time, and the longitudinal effects of online flow on IA symptom severity between the ages of 16 and 18 years.

To address these aims, the present study adopted a comprehensive conceptual framework of understanding IA (Stavropoulos, Kuss, Griffiths \& Motti-Stefanidi, 2016b). This framework views IA symptoms along a continuum (ranging from minimum to maximum severity) and emphasizes the interplay between 'push' and 'pull' factors over 
the psychosocial development of the Internet user. Push factors include age-related changes (maturation), gender, characteristics of the individual and factors within the individual's close personal context, which may contribute to their vulnerability to IA (Anderson et al., 2016). Indicatively, past empirical studies have illustrated among others the significance of low conscientiousness and the presence of obsessive-compulsive and anxious manifestations as individual level push factors, as well as the higher average levels of classroom hostility and extraversion as contextual level push factors (Stavropoulos, Kuss, Griffiths \& Motti-Stefanidi, 2016b; Stavropoulos, Gentile \& MottiStefanidi, 2016b; Stavropoulos et al., 2017b). Pull factors entail characteristics related to communicative features of the Internet, that may attract the user to the context of the medium (Jones, Scholes, Johnson, Katsikitis, \& Carras, 2014). Past empirical findings have identified online flow as an important IA pull factor; as online flow defines how absorbed the individual becomes in the online activity and the preference in playing Massively Multiplayer Online Role Playing games, thus highlighting its importance (Stavropoulos, Alexandraki \& Motti-Stefanidi, 2013b; Stavropoulos, Kuss, Griffiths, Wilson \& Motti-Stefanidi, 2017a). Furthermore, literature has suggested that associations between aspects of IA (including low social involvement and low sense of wellbeing) and various Internet-related factors (such as online communication usage) may alternate or even reverse over the course of adolescent development and as a function of individual characteristics (such as introversion and extroversion; Kraut et al., 2002). In addition, previous cross-sectional research supports associations between gender and humancomputer interaction concepts, such as online flow (Stavropoulos et al., 2013ab). The aim of the present study was to extend this work by examining the moderating effects of agerelated changes on the associations between gender and online flow and IA symptoms.

\subsection{Age-Related Changes}


Adolescents appear to be at relatively high risk for developing IA (Kuss et al., 2014; Stavropoulos et al., 2013a). During adolescence, individuals seek more private space to accommodate their emerging adult needs (Kagitcibasi, 2013). Furthermore, the closer adolescents get to becoming adults, the higher their drive for independence becomes (Dumas, Ellis, \& Wolfe, 2012; Kagitcibasi, 2013). This developmental transition may often be facilitated by the independence/privacy that adolescents experience online, which in turn might lead to IA (Kuss et al., 2014). In addition, tensions related to identity formation processes, challenging interpersonal links and lack of cohesiveness in adolescent behavior (Rohde, Lewinsohn, Klein, Seeley \& Gau, 2013) have been suggested to increase addiction risk in general (Dumas et al., 2012) and IA risk in particular (Kuss et al., 2014). Counterintuitively, maturational and socialization processes (Blakemore, \& Mills, 2014) occurring during adolescence, would enable individuals to be progressively more equipped to address their stressors without escaping online (e.g., using higher cognitive skills such as attention focusing, abstract thinking, social reasoning, and advanced thought processing), and therefore to reduce their IA symptoms. Brain (prefrontal cortex) and executive functions' development occuring in late adolescence could also result in increased inhibition, which is a key skill necessary to moderate addictive manifestations in general and addictive Internet use specifically (Bernheim, Halfon, \& Boutrel, 2013; Steinberg, 2007). This is in accordance with other work supporting that the level of various addictive motives such as hedonism, nurturance (Haylett, Stephenson \& Lefever, 2004; Mac Laren \& Best 2010), and escapism (Kuss et al., 2012) also vary over the course of development (Foddy \& Savulescu, 2010) and that other addictive behaviors (e.g., binge drinking, illicit drug use) decrease during the transition from adolescence to young adulthood (Foster, Hicks, Iacono, \& McGue, 2015). Based on the above, it could be hypothesized that in adolescence, IA symptom severity is 
susceptible to age-related effects, with severity being higher in earlier developmental stages (due to developmental challenges), and lower during later developmental stages (due to maturation processes). Nevertheless, age related effects on IA behaviors during adolescence have been predominantly examined by cross-sectional studies, with some indicating no significant effects of age (Poli \& Agrimi, 2012), others suggesting that older adolescents present with higher IA risk (Karacic \& Oreskovic, 2017), and the majority of findings concluding that IA behaviors tend to decrease during adolescence (Yen, Ko, Yen, Chang \& Cheng, 2009; Bakken, Wenzel, Götestam, Johansson \& ØREN, 2009). In that line, and given the significance of the age of onset/ initiation of addictive behaviors in general for their future prognosis (Beard, Haas, Wickham \& Stavropoulos, 2017), the past contradictory findings (Poli \& Agrimi, 2012; Karacic \& Oreskovic, 2017; Yen et al., 2009; Bakken et al., 2009) and the limitations of applying cross-sectional designs to assess maturation effects (e.g. different time cohorts may present with different growth patterns over the same period of development; Motti-Stefanidi \& Masten, 2017); the present study is (to the best of the authors' knowledge) the first that aimed to assess this question by implementing a longitudinal design, whilst specifically investigating the time period between 16 and 18 years.

Studies have repeatedly highlighted the significance of the period between 16 and 18 years for the development of IA symptoms (e.g., Stavropoulos et al. 2017ab; Stavropoulos et al., 2016a, 2016b). This period sets in place patterns of behavior that may lead to high Internet use (Society of Information Observatory, 2010; Van Deursen \& Van Dijk, 2014). This developmental time also overlaps with the period in which potentially high-risk applications (e.g., gaming and social networking sites) are used most (Society of Information Observatory, 2010), and it coincides with an educational transition to elective 
school attendance (rather than mandatory attendance) within the educational system of some countries (including Greece, where the data for this study were collected) (Stavropoulos et al., 2017). The increased developmental significance of the time between 16 and 18 years for the establishment of addictive behaviors in general, along with the coexistence of increased IA risk factors (use of high risk Internet applications) and possibly protective age-related/maturation effects, make this developmental time period (between 16 and 18 years) a valuable period for testing the hypothesis (that IA symptoms severity would be susceptible to age-related effects, with IA severity being higher earlier and lower during later adolescence).

\subsection{Gender}

Alongside age-related changes between 16 and 18 years, gender-related changes in various behaviors as well as differences in experiencing IA symptoms have been investigated (e.g., De Bolle et al., 2015, Stavropoulos et al., 2013a, 2013b). More specifically, longitudinal findings have shown that males are more inclined to develop problems with impulse-control and sensation-seeking behaviors than females, and these symptoms have been associated with IA (Kuss et al., 2014; Shulman, Harden, Chein, \& Steinberg, 2015). Additionally, cross-sectional studies of Internet use have indicated adolescent males being at higher risk of IA than adolescent females (Shi, Wang, \& Zou, 2017; Karacic \& Oreskovic, 2017; Ostovar et al., 2016; Anderson et al., 2016; Chen et al., 2015; Choo et al., 2015; Gentile et al., 2011; Haagsma et al., 2013; Hong et al., 2014; Willoughby, 2008; Yu \& Shek, 2013; Stavropoulos et al., 2013a; Yen et al., 2009; Shaw \& Black, 2008; Johansson \& Götestam, 2004). Higher digital skills and preference for more addictive applications (such as gaming) have been suggested to explain these findings (Kuss \& Griffiths, 2012). Despite these, some longitudinal studies did not 
support any gender-linked differences considering IA related behaviors (Gámez-Guadix, 2014; Gámez-Guadix et al., 2015; Jackson et al., 2003).

Overall though, it has been acknowledged that there is a dearth of longitudinal research considering gender-related variations in IA symptoms over the life span, during adolescence in general, and the time period between 16 and 18 years in particular (Anderson et al., 2016). This is important provided that gender-related trajectories (with males being at higher risk) have been demonstrated in relation to other forms of addictive behaviors (e.g., illicit drug use) during the transition from adolescence to young adulthood (Foster et al, 2015). Gender differences appear to increase during late adolescence (16-18 years) in major areas of development (e.g., romantic life), with males presenting gradually more relationship avoidance, exhibiting less elaborate social skills, and adopting less communicative coping strategies than females (Del Giudice, 2011; Williams, 2015). Such differences in relationship avoidance and social skills in general could indeed increase IA symptoms severity for males, provided that IA has been potentially considered as a manifestation of avoidant behavior indicative of low social skills (Kuss et al., 2014).

\subsection{Online flow}

In addition to age and gender differences in IA, online flow has been found to contribute to the development of IA symptoms (Stavropoulos et al., 2013b). Flow is a positive psychology concept (Csikszentmihalyi, 1997) that has been integrated into the IA field (Chen, Wigand \& Nilan, 1999; Hoffman \& Novak, 2009). Flow is described as a state that occurs whilst using the Internet and it entails a seamless sequence of responses (active engagement of the user to the challenging and inviting elements of Internet activities) facilitated by machine interactivity. Furthermore, being in a state of flow is intrinsically enjoyable and self-reinforcing, while it is combined with a loss or decrease of self-consciousness (Hoffman \& Novak, 2009). At this point it it should be noted that in 
line with the above, the international literature has consistently conceptualized flow as a precursor of IA behaviors and not the opposite (Anderson et al., 2016; Stavropoulos et al., 2013; Douglas et al., 2008; Kim \& Davis, 2009; Rettie, 2001). Particulalry, it has been postulated that the experience of online "flow", as an emotional state, defines the quality/intensity (e.g., absorbance, length of time and the level of disconnection from the offline world) of Internet use, and therefore, the potential emergence of IA behaviors (e.g., it is the level of flow experience that may result to IA and not the other way around; Anderson et al., 2016; Stavropoulos et al., 2013; Douglas et al., 2008; Kim \& Davis, 2009; Rettie, 2001).

In that line, there appear to be significant findings concidering the association of online flow and IA (Stavropoulos et al., 2013b). Given the relationship between online flow and gratification (Hoffman \& Novak, 2009) and the role of the latter as a precipitating and perpetuating factor in the development of addictions (Leung, 2014), it is assumed that adolescent users who experience more online flow are at higher risk for IA. This hypothesis is supported by a number of recent cross-sectional studies (Yang, Lu, Wang, \& Zhao, 2014; Stavropoulos et al., 2013b). However, provided that levels of executive functions involved with flow experiences increase over time during late adolescence, it is likely that the association between online flow and IA symptoms severity would vary between 16 and 18 years (Bernheim et al., 2013; Stavropoulos et al., 2013b; Steinberg, 2007).

\subsection{The Present Study}

This research adopted a dimensional (continuum of minimum to maximum severity) conceptualization of IA (Stavropoulos et al., 2016a) to enable the examination of the whole spectrum of variations of IA symptoms. This dimensional approach on IA has been widely followed by international researchers and the measurements used to support it 
have been validated in Greek populations, such as the sample of the present study (Kuss et al., 2014; Stavropoulos et al., 2013b).

To study the whole range of severity of IA behaviors, a suggested comprehensive conceptual framework of understanding IA was applied (Stavropoulos et al., 2016a). This acknowledges the distribution of IA symptoms along a continuum and highlights the importance of the interplay between "push" (factors that increase the user's vulnerability to IA) and "pull" (factors that increase the addictive features of the Internet) factors over the psychosocial development of the Internet user. To appropriately investigate the effects and the interactions of push and pull factors across each of the levels involved (agerelated, individual and contextual changes), this framework is best employed using multiple levels of analyses that disentangle the effects related to the developmental stage, the person and the person's context (Stavropoulos et al., 2016a, 2016b).

Accordingly, the present longitudinal study examined a sample of Greek adolescents (assessed at 16 and again at 18 years of age) to determine the effects of individual level push and pull factors in regards to the whole range of IA symptom severity. Two levels of analysis were applied: Level 1 was used for temporal factors (i.e., age-related changes in IA), and Level 2 was used to study one individual push factor (i.e. being male) and an individual pull factor (i.e., online flow). Random effects on IA symptoms due to clustering of the participants were controlled in the third level of the model applied. Based on relevant literature and to expand the extant knowledge in the field, the following specific research hypotheses were formulated:

$H_{1}$ : It was hypothesized that IA symptoms decrease between the ages of 16 and 18 years. Maturational and socialization processes developing during adolescence (Bernheim et al., 2013; Blakemore, \& Mills, 2014; Steinberg, 2007), would enable Internet users to 
become gradually better at coping with their real-life stressors over time without requiring to use the Internet as a coping mechanism to avoid their problems.

$H_{2}$ : It was hypothesized that males present higher IA symptoms compared to females between 16 and 18 years. Higher digital skills of males and their preference for more addictive applications (Kuss \& Griffiths, 2012) can result in higher IA severity. Furthermore, it is expected that IA symptom severity would increase more for males compared to females over time. Gender differences appear to increase during late adolescence (16-18 years) in critical areas of development (e.g., romantic life), with males being less socially skilled and more relationship-avoidant than females (Del Giudice, 2011).

$H_{3}$ : Based on the association between online flow and gratification (Stavropoulos et al., 2013b) and the causal role of gratification as a precipitating and perpetuating factor in the development of addictions (Leung, 2014), it was hypothesized that adolescents who experience more online flow present higher IA symptoms.

\section{Method}

\subsection{Participants}

The present paper utilized longitudinal data collected in Greece ${ }^{1}$. The data were collected through a paper-pencil process during two school teachning hours that was first approved by the Greek Ministry of Education, then by the Teachers' Council of each school, and finally by the parents/guardians of the participants. The sample was chosen in the capital metro area and a selected regional area (Korinthia, Greece) using the method of randomized stratified selection (Kernan, Viscoli, Makuch, Brass \& Horwitz, 1999) in accordance with the inventory card of the Ministry of Education. The ratios of high schools and students were estimated between these areas as well as between academic and 
vocational track high schools within them. Based on these quotas, school units and participants were selected via lottery.

The sample comprised 648 students embedded in 34 classrooms of public, academic and vocational track high schools ${ }^{2}$. Chi-squared analysis confirmed that the distribution of the included sample did not differ significantly from that of the original population considering the area of residence and the type of school attended $\left(X^{2}=3.83\right.$ $p=.75)($ see Table 1). The estimated maximum sampling error with a sample size of 648 was 3.85 at the $95 \%$ confidence level $(Z=1.96)$. All the schools elected for participation took place in the first wave. Parents' consent was $98 \%$ and the students' response rate was over $95 \%$. This did not significantly vary between diffrenet classrooms and types of schools at Time 1.

-Table 1. Sample and Population Proportions-

With respect to the parents' and guardians' socioeconomic profile, $78.7 \%$ were married, $8.3 \%$ of the mothers and $8.6 \%$ of the fathers were unemployed, and $89 \%$ of the mothers and $87 \%$ of the fathers had completed high school. At Time 1, internet usage was a $100 \%$, with $21 \%$ using predominantly blogs and social networking sites, $16.4 \%$ instant messengers, $14.6 \%$ information seeking engines, $13.6 \%$ chatrooms, $13.4 \%$ online games, 13.4\% you-tube and videos, $2.4 \%$ pornographic sites, and 5\% other applications. Parents' consent was $98 \%$ and the students' response rate was over $95 \%$. The estimated maximum sampling error with a sample size of 648 is $3.85 \%$ at the $95 \%$ confidence level $(Z=1.96)$.

The cohort was assessed twice in a period of two school years (wave 1: Mean age $=$ 15.75 years, $S D=0.57$ years, boys $=301,46.4 \%$, girls $=347,53.6 \%$, Academic Track High Schools $=540,83.2 \%$, Vocational Track High Schools $=108,16.7 \%$, Athens Metro Area $=$ $600,92.6 \%$, Korinthia $=48,7.4 \%$; Wave $2:$ Mean ${ }_{\text {age }}=17.75$ years, $S D=0.54$ years, boys $=$ 
181, 49.9\%, girls $=182,50.1 \%$, Academic Track High Schools $=292,80.3 \%$, Vocational Track High Schools $=71,19.7 \%$, Athens Metro Area $=326,89.9 \%$, Korinthia $=37,10.1 \%$; Group-classroom level controlled for waves 1\&2: 1 classroom, 2.9\%, Vocational Track Korinthia, 3 classrooms, 8.8\% Academic Track Korinthia, 5 classrooms, 14.7\% Vocational Track Athens Metro Area, 25 classrooms, 73.5\% Athens Metro Area). Retention between the two waves was $56 \%\left(N_{\text {Wave } 2}=363\right)$ due to changes of school, school, and research drop-outs. The frequency of assessments for each individual varied $(1-2, M=1.57)$. Although attrition was unsystematic, attrition effects were evaluated. Specifically, attrition was used as an independent variable (dummy coded $0=$ Retention, $1=$ Attrition) to assess whether it correlated with IA scores and whether it interacted with the independent variables (e.g., time, gender, online flow) on IA symptoms. Findings revealed that attrition was not associated with IA symptoms and neither did it affect the independent variables' associations with the dependent variables used in the model (see Table 2).

-Table 2. Assessment of the Effects of Attrition in HLM analysis -

\subsection{Instruments}

In the paper-based survey, independently trained researchers collected the data as part of a wider study on adolescent Internet use and abuse in Greece ${ }^{1}$.

Internet Addiction Test : The Internet Addiction Test (IAT), as probably the most widely used test internationally, was used to assess IA symptoms from minimum to maximum (Stavropoulos et al., 2013a, 2013b; Young, 1998). The IAT comprises 20 questions evaluating negative consequences of excessive Internet use. Questions 2, 3 and 8 were appropriately modified to reflect age/adolescence-related content (i.e., in Question 8, "job performance" was replaced by "school performance" turning into "How often does your school performance or productivity suffer because of the Internet?’). Questions used 
a Likert scale format ( $1=$ "not at all" to $5=$ "always", and $0=$ "does not apply"). Scores from each question are added together with total scores ranging from $0-100$, with 0 indicating minimum and 100 indicating maximum IA symptoms. IAT adaptation in a normative sample of Greek adolecents revealed a score of 50 as the cut off point for IA (Stavropoulos et al., 2013a). The instrument presented high concurrent validity in previous studies, resulted to an optimum cut-off point of 51 for detecting IA among Greek adolescents (Stavropoulos et al., 2013a), and its internal reliability in the present study was high $($ Cronbach alpha $[\mathrm{CA}]=0.93)$.

Online Flow Questionnaire: To assess Internet flow, the Online Flow Questionnaire (OFQ; Chen, Wigand \& Nilan, 1999) was used after bidirectional translation from bilingual translators. The OFQ comprises five pairs of self-reported questions relating to flow experience. Respondents were first asked whether they had ever had a flow experience in the Internet environment $(0=$ No, $1=$ Yes $)$ as described by each question (e.g., 'Have you ever experienced the feeling of 'positive challenge' during your Web navigation?'). Secondly, participants were asked to define the application in which they had experienced it. For the final score, the numbers of positives answers in the first question of each of the five pairs were added together. Therefore, online flow scores ranged from $0-5$, with 0 indicating minimum and 5 indicating a maximum rate of online flow experiences. The reliability co-efficient of the present version of the questionnaire was acceptable with a Kuder-Richardson of 0.70 and similar to that revealed in previous Greek studies (Stavropoulos et al., 2013b).

\subsection{Procedures}

The first time point assessments were collected in the school year 2009-2010 and the second time point assessments were collected in the school year 2011-2012. The 
process of data collection was identical between the two time points. A specially trained research team of 13 undergraduate, postgraduate, and PhD students of the Department of Psychology of the University of Athens collected the data in the participants' classrooms during the first two or the last two school hours (45 minutes each) of a school day, according to the permission provided by the Ministry of Education. The adolescents were motivated to participate in the study by the fact that they would not have to attend subjects taught during the time of the study and they would not be considered as absent from lessons. It should be noted that according to the Greek school regulation, students are allowed to progress to the next grade on the condition that they have not exceeded 50 school hours of unjustified absence per school year.

\subsection{Analyses}

Multilevel modeling was used to statistically analyze a data structure where measurements at two time points (Level 1) were nested within individuals (Level 2), who were nested within classrooms (Level 3) $)^{2}$. It should be highlighted that given the theoretical conceptualization of online flow as a causative factor of IA behaviors and not the reverse, the use of cross-lagged analyses was not selected (Anderson et al., 2016; Stavropoulos et al., 2013; Douglas et al., 2008; Kim \& Davis, 2009; Rettie, 2001). Furthermore, the multilevel modeling approach was chosen to enable the study to disentangle and examine age-related changes on IA symptoms at Level 1 and the effects of the individual push factor (i.e., being male) and the individual pull factor (i.e., online flow) on IA symptoms at Level 2, while controlling for possible random effects due to the nesting-clustering of the data (participants within classrooms) at Level 3. Subsequently, HLM 6.0.8 software was used ${ }^{3}$ (Raudenbush \& Bryk, 2002). Model testing proceeded in successive phases, such that each of the examined conditions were first studied separately, 
before included in the full model (Hox, 2010; Raudenbush \& Bryk, 2002): 1)

Unconstraint (null) model; 2) Random ancova model (Level 1 predictor); 3) Means as outcomes model (Level 2 predictor-gender); 4) Random coefficient (regression slope) model (Levels 1 \& 2 predictors-time and gender); 5) Means as outcomes model (Level 2 predictor-flow); 6) Random coefficient (regression slope) model (Levels $1 \& 2$ predictorstime and flow) and 7) The full model (Level 1 and Level 2 predictors-time, gender and flow). Due to the results not being statistically significantly different, only the full model will be reported here. In this context, IA symptoms (Level-1outcome variable) were predicted for each individual at Level 1 by wave in the study. Wave was centered at wave 1 such that the individual intercepts referred to the initial Level of ARA (Wave $1=0$, Wave 2=1). The individual initial Level and the individual linear change over the two assessments (slope) were predicted at Level 2 by gender and online flow. Gender was centered on girls $(0=$ girls, $1=$ boys $)$. Finally, random effects due to the clustering of the participants were controlled through random effects equations at Level 3 in regards to both the main effects of time and gender, as well as the cross-level interactions of time with gender and online flow (slopes). To control for mis-specification (i.e., lack of linearity) and the distributional assumptions at each level (lack of normality, heteroscedacity), HLM results accounting for robust standard errors (which are insensitive to possible violations of these assumptions) were calculated. Considering missing values, whereas they do not present a problem at Level 1 in HLM and did not occur at Level 3 (classrooms), missing values at Level 2 (individuals) were addressed. Although they were unsystematic, to avoid list-wise deletion, multiple imputation was applied (five Maximum Likelihood imputations using SPSS) using all available Level 2 variables. This type of imputation was selected as it outperforms listwise deletion for parameters involving many recouped cases and results to improve standard error estimates (Newman, 2003). Based 
on previous literature, all multilevel analyses were calculated five times and their results were averaged (Motti, Asendoprf \& Masten, 2012).

\section{Results}

Before proceeding with the main HLM analyses, to address the theoretical hypostheses introduced, the prevalence of IA was calculated across the two waves based on the optimum cut-off point of 51 previously suggested as applicable for detecting IA among Greek adolescents (Stavropoulos et al, 2013). Based on this cut-off point, the IA prevalence was $2 \%$ (13 participants in time point 1$)$ and $0.8 \%$ (3 participants at time point 2).

Prior to the HLM analyses, the means, standard deviations and inter-correlations between the HLM variables were estimated (see Table 3).

-Table 3. Means, standard deviations and correlations of the HLM variables -

To assure that the three levels contributed to variation in IA scores, the level components were calculated from the unconditional model $\left(X^{2}\right.$ Level 2=1272.85, $\mathrm{df}=596$, $\mathrm{p}=.001, \mathrm{x} 2$ Level $3=46.93, \mathrm{df}=33, p=.001)$. As an additional step, the intra class correlation (ICC) was calculated to determine which percentage of the variance in IA is attributable to classroom membership (Level 3), which percentage is attributable to between individual differences (Level 2) and which to over-time differences within individuals (Level 1). Results suggested that 57\% (180.26) of the variance in IA is at the first Level (over time differences within individuals), 41\% (131.75) at Level 2 (the individual level) and 2\% (5.82) at Level 3 (between classrooms-controlled in the present analyses). 
Therefore, HLM equations were calculated (see Appendix). The Level 1 intercepts for the cross-sectional findings were 29.40 for females and 29.44 for males. These were the estimated mean IA scores for adolescents of average online flow at Time 1. Considering how IA symptoms develop between 16 and 18 years, the time coefficient was $b=-7.16(p=.001)\left(H_{1}\right)$. Consequently, the average IA score of adolescents at the age of 18 years dropped by 7.16. In regards to how gender (being male) contributes to the change of IA symptoms between 16 and 18 years, the interaction coefficient of time at Level 1 and being male at Level 2 was $b=5.03(p=.016)\left(H_{2}\right)$. This indicated that the average drop of IA symptoms between 16 and 18 years was lower for boys than for girls $(7.16-5.03=2.13)$.

Considering how online flow and IA symptoms were associated with each other at Time 1, the online flow coefficient was $b=5.06(p=.001)\left(H_{3}\right)$. Consequently, the average IA score of adolescents who scored one point higher than the estimated mean in online flow increased by 5.06. Considering the effect of online flow at Time 1 on IA at Time 2, the interaction coefficient of online flow with time was $b=.16,(p=.852)$, indicating that the effect of online flow on IA symptoms did not interact with time significantly. The complete model explained $17 \%$ of the Level 1 variance $(9.69 \%$ of the three levels' variance) and $25 \%$ of the Level 2 variance $(10.25 \%$ of the three levels' variance). Considering the overall IA variance, the model explained $19.94 \%$. Analyses controlled for random effects due to other individual level random effects and clustering.

-Table 4. HLM Analysis Predicting Adolescents' Internet Addiction Scores -Figure 1. Model -

-Figure 2. IA Symptoms according to online flow- 


\section{Discussion}

The present longitudinal study investigated IA symptoms on a continuum of severity in a normative sample of Greek adolescents enrolled in public high schools. A comprehensive conceptual framework (Stavropoulos et al., 2016a) was adopted to examine age-related effects in conjunction with individual level "push" factors (that increase vulnerability to IA) and "pull" factors (that increase the addictive features of Internet activities). Specifically, being male was examined as an individual level "push" factor and online flow was examined as an individual level "pull" factor in conjunction with age-related changes during late adolescence (16-18 years). A three-level hierarchical linear model was applied to analyze IA symptoms severity change between 16 and 18 years in respect to gender and online flow, controlling for random effects in relation to the clustering of the adolescent students. Results showed that IA symptoms decreased between 16 and 18 years, and the decrease was lower for males. Online flow was associated with higher IA symptoms and this relationship did not vary significantly over time. The implications of these findings for the understanding, prevention and treatment of IA symptoms in adolescence are discussed below.

\subsection{Age-related variations in understanding, prevention and treatment of}

\section{IA symptoms}

IA symptoms decreased between the ages of 16 and 18 years, suggesting that IA vulnerability diminishes after the age of 16 years. This is consistent with other work showing that the level of various addictive motives including hedonism, nurturance (Haylett, Stephenson \& Lefever, 2004; Mac Laren \& Best, 2010) and escapism (Kuss et 
al., 2012) change over the course of development (Foddy \& Savulescu, 2010), and that addictive behaviors (e.g., illicit drug use, binge drinking) decrease between adolescence and young adulthood (Foster et al, 2015). As adolescence is a transitional stage (Blakemore, \& Mills, 2014), developmental drives that may contribute to IA symptoms could be either satisfied in other ways or even de-escalate over time. It is possible that such drives and needs for interactivity, marking a transitional period in development (Blakemore, \& Mills, 2014), may be later expressed more adaptively through off-line social activity rather than online. An Internet "satiation" period similar to other forms of addictions (Sussman \& Sussman, 2011) has been proposed and is a concept requiring further investigation. In short, the present findings suggest that after an acute engagement period before the age of 16 years, cravings for Internet use may decline, and become replaced by more effective internal controls due to maturational and socialization processes.

Our results imply that IA symptoms function as a developmental trajectory rather than an "addiction" or "clinical problem" at the age of 16 years. The development of prefrontal executive functions' during late adolescence is known to enhance inhibitory control, perhaps providing adolescent Internet users with the capacity to moderate addictive/excessive Internet use (Bernheim et al., 2013; Steinberg, 2007). Despite the need for more longitudinal and cross-cultural studies of typical adolescent development between 16 and 18 years, our results have direct implications for planning prevention and treatment initiatives. More specifically: (i) the need for more prevention resources and programs to be allocated to adolescents before the age of 16 years in order to prevent agerelated IA behaviors from escalating into clinical problems later in life - especially for more vulnerable individuals; (ii) although many IA-related behaviours decrease over time 
as a natural course of adolescent development there can still be significant harmful consequences of IA from a clinical perspective during this period (e.g., lack of relationships, isolation, academic decline, decreased wellbeing; Anderson et al., 2016). Therefore, and despite the average decrease of IA behaviours over time, younger individuals at-risk need to be protected in advance from the potential consequences of IA (Stavropoulos et al., 2017ab) and; (iii) the need for intervention and treatment programs (when there are limited resources, and priorities need to be defined) to target Internet users who present with severe IA symptoms after 18 years, posing a clinical risk.

\subsection{Gender differences in understanding, prevention and treatment of IA} symptoms

Similar to previous cross-sectional studies, males presented with higher IA symptom severity at both 16 and 18 years (although the difference was not statistically significant at 16 years) (Shi et al., 2017; Karacic \& Oreskovic, 2017; Ostovar et al., 2016; Anderson et al., 2016; Chen et al., 2015; Choo et al., 2015; Gentile et al., 2011; Haagsma et al., 2013; Hong et al., 2014; Willoughby, 2008; Yu \& Shek, 2013; Stavropoulos et al., 2013a, 2013b; Yen et al., 2009; Shaw \& Black, 2008; Johansson \& Götestam, 2004). Furthermore, IA symptoms decreased over time in both males and females, with the decrease lower for males. Adolescent boys appear to be more oriented to digital media than girls and usually engage in more absorbing Internet applications such as gaming (Kuss \& Griffiths, 2012). Additionally, males are at higher risk for addictive behaviors in general (Crane Langenecker, \& Mermelstein, 2015). Less developed social skills, higher relationship avoidance, and less communicative responses to stress in males (in the context of everyday interaction) may enhance the likelihood of excesive online socialization in males compared with females over time, and thus potentially maintaining 
higher levels of IA symptoms (Del Giudice, 2011). To our knowledge, although a higher IA risk for males has been shown repeatedly in cross-sectional studies, the present finding is perhaps the first to clarify the nature of IA gender differences longitudinally (Shi et al., 2017; Karacic \& Oreskovic, 2017; Ostovar et al., 2016; Anderson et al., 2016; Chen et al., 2015; Choo et al., 2015; Gentile et al., 2011; Haagsma et al., 2013; Hong et al., 2014; Willoughby, 2008; Yu \& Shek, 2013; Stavropoulos et al., 2013a, 2013b; Yen et al., 2009; Shaw \& Black, 2008; Johansson \& Götestam, 2004).

This result implies gender differences in IA tend to progressively increase during late adolescence, becoming more prominent later in development. This accords with findings that reveal gender-related differences emerge more strongly later in adolescence in other forms of psychopathology and addiction-illiciting substance use (Crane et al., 2015; Foster et al, 2015). IA risk factors, including lower social skills, higher relationship avoidance and less communicative responses to stress, increase during the transition from adolescence to adulthood during late adolescence, and could gradually elevate IA risk for males (Del Giudice, 2011; Williams, 2015). Although further longitudinal and crosscultural studies are required to support these explanations, directions for IA prevention and treatment are implied. Prevention initiatives should embrace an element of gender differentiation progressively adjusting their emphasis (during the period between 16 and 18 years) to the higher risk of males (e.g., increasing awareness about male preferences to high risk internet appliations).

\subsection{Online flow in understanding, prevention and treatment of IA symptoms}

In relation to online flow, the results demonstrated that higher online flow scores were associated with higher IA symptoms. The association between online flow and IA confirms several cross-sectional findings (Yang et al., 2014; Stavropoulos et al., 2013b), 
and highliths online flow's link to online satisfaction (Stavropoulos et al., 2013b; Hoffman \& Novak, 2009; Zaman et al., 2010). Since addictive behaviors have been associated with gratification (Sussman \& Sussman, 2011), higher levels of flow (and thus online gratification) may place adolescents at higher risk for IA.

A novel finding was that the strength of the contribution of online flow to IA symptoms did not vary as a function of age. This is in line with the international literature that suggests that the intensity of flow experiences may not vary across different age groups (Csikszentmihalyi, 1997) and indicate how the concenpt of online flow could be embraced in the planning of IA prevention and intervention initiatives. In particular, IA prevention programs in adolescence could include psychoeducation activities in regards to online flow (e.g., explaining how the interactions with online applications become intrinsically enjoyable and self-reinforcing, combined with a dampening of selfconsciousness); this might reinforce the individuals' level of IA risk awareness and selfreflection (Hoffman \& Novak, 2009). In particular, counselling related reflections and questions targeting the absorbing effect of flow, in order to raise awareness could be potentially informed by the present findings (e.g., how can online engagement result to disengagement in real life; online pleasure as a source of offline unhappiness; time flowing online and stagnating in real life).

\subsection{Limitations and further research}

The current study has some limitations that do bear on its interpretation. First, selfreport assessments were used which rely on the participants' perception and disposition to answer genuinely, and therefore might reduce the validity and the reliability of the findings. Furthermore, due to the specific age and cultural background of the sample, some caution is warranted when generalizing to populations of different developmental 
stages and cultural heritage. Additionally, the retention rate of $56 \%$, although having been assessed and not being rare in the context of prospective studies, needs to considered as a potential limitation in the interpretation of the present findings. In that context, the period that mediated the collection of the data and the completion of the present study needs to be considered as an additional limitation. It is noted that developments in internet applications and changes in the trends of Internet use, that may have occurred during this time, could impact the generalizability of the findings (Anderson et al., 2016). Finally, additional parameters of online engagement should be taken into account (e.g., time spent online per day or week, and preferred Internet application). Moreover, the interplay between characteristics of the individuals and elements of their real and virtual context regarding IA should be explored. Understanding the dynamic interplay of such factors will enable researchers to build a broader theory of IA during adolescence.

Notwithstanding these limitations, our longitudinal examination of age-related changes in online flow is a unique contribution to the literature. Unique findings include those showing IA symptoms to be a function of a developmental trajectory, the increasing IA risk for males and its resilience to age-related effects, and the contribution of online flow to IA symptoms severity between 16 and 18 years.

\subsection{Conclusion and implications}

Research on IA has made important advancements in the last two decades, offering significant new knowledge concerning risk and protective factors. However, there has been a dearth of longitudinal studies, as well as studies related to human computer interaction concepts associated with IA symptoms. The present study highlights the need for considering online flow as significant IA risk (pull) factor between the ages of 16 and 18 years. Furthermore, the present findings indicate that higher IA behavior at 
the age of 16 years could be additionally evaluated within a developmental framework, with gender differences widening over time.

The findings of the present study have several important implications. Prevention initiatives and programs could prioritize adolescents before the age of 16 years, embrace an element of gender differentiation (during the period between 16 and 18 years) to address the progressively higher IA risk in males, and include psychoeducation regarding the effects of online flow on IA symptoms severity.

\section{References}

Anderson, E. L., E. Steen, and V. Stavropoulos. (2016). "Internet use and Problematic Internet Use: A systematic review of longitudinal research trends in adolescence and emergent adulthood." International Journal of Adolescence and Youth 1: 1-25. doi:10.1080/02673843.2016.1227716 
Asendorpf, J. B., and M. A. Van Aken. (2003). "Validity of Big Five personality judgments in childhood: A 9 year longitudinal study." European Journal of Personality 17(1): 1-17. doi:10.1002/per.460

Bakken, I. J., H. G. Wenzel, K. G. Götestam, A. Johansson, and A. Øren. (2009). “Internet addiction among Norwegian adults: A stratified probability sample study." Scandinavian Journal of Psychology 50(2): 121-127. doi: 10.1111/j.14679450.2008.00685.x

Baumgartner, H., and C. Homburg. (1996). “Applications of structural equation modeling in marketing and consumer research: A review." International journal of Research in Marketing 13(2): 139-161. doi:10.1016/0167-8116(95)00038-0

Beard, C. L., A. L. Hass, R. E. Wickham, and V. Stavropoulos. (2017). “Age of initiation and Internet Gaming Disorder: The role of self-esteem." Cyberpsychology, Behavior, and Social Networking 20 (6): 397-401. doi:10.1089/cyber.2017.0011

Bernheim, A., O. Halfon, and B. Boutrel. (2013). "Controversies about the enhanced vulnerability of the adolescent brain to develop addiction." Frontiers in Pharmacology 4: 118. doi:10.3389/fphar.2013.00118

Blakemore, S. J., and K. L. Mills. (2014). "Is adolescence a sensitive period for sociocultural processing?" Annual Review of Psychology 65: 187-207. doi:10.1146/annurev-psych-010213-115202

Chen, H., R. Wigand, and M. S. Nilan. (1999). "Optimal experience of web activities." Computers in Human Behavior 15: 585-608. doi:10.1016/S0747-5632(99)00038-2 
Chen, Y. L., S. H. Chen, and S. F. S. Gau. (2015). “ADHD and autistic traits, family function, parenting style, and social adjustment for Internet addiction among children and adolescents in Taiwan: A longitudinal study.” Research in Developmental Disabilities 39: 20-31. doi:10.1016/j.ridd.2014.12.025

Choo, H., T. Sim, A. K. F. Liau, D. A. Gentile, and A. Khoo. (2015). "Parental influences on pathological symptoms of video-gaming among children and adolescents: A prospective study." Journal of Child and Family Studies 24: 1429-1441. doi:10.1007/s10826-014-9949-9

Coie, J. D., K. A. Dodge, and H. Coppotelli. (1982). "Dimensions and types of social status: A cross-age perspective.” Developmental Psychology 18(4): 557. doi:10.1037/0012-1649.18.4.557

Crane, N. A., S. A. Langenecker, and R. J. Mermelstein. (2015). "Gender differences in the associations among marijuana use, cigarette use, and symptoms of depression during adolescence and young adulthood." Addictive Behaviors 49: 33-39. doi:10.1016/j.addbeh.2015.05.014

Csikszentmihalyi, M. (1997). Finding flow: The psychology of engagement with everyday life. New York: Basic Books.

De Bolle, M., F. De Fruyt, R. R. McCrae, C. E. Löckenhoff, P. T. Costa Jr., M. E. AquilarVafaie, C. K. Ahn, H. N. Ahn, et al. (2015). "The emergence of sex differences in personality traits in early adolescence: A cross-sectional, cross-cultural study." Journal of Personality and Social Psychology 108(1): 171-185. doi:10.1037/a0038497 
Del Giudice, M. (2011). "Sex differences in romantic attachment: A meta-analysis." Personality and Social Psychology Bulletin 37(2): 193-214. doi:10.1177/0146167210392789

Derogatis, L. R., and K. L. Savitz. (1994). The SCL-90-R, Brief Symptom Inventory and matching clinical rating scales: The use of psychological testing for treatment planning and outcome assessment (2nd ed.). Hills-dale, NJ: Lawrence Erlbaum.

Douglas, A. C., J. E. Mills, M. Niang, S. Stepchenkova, S. Byun, C. Ruffini, S. K. Lee, et al. (2008). "Internet addiction: Meta-synthesis of qualitative research for the decade 1996-2006." Computers in Human Behavior 24(6): 3027-3044. doi:10.1016/j.chb.2008.05.009

Dumas, T. M., W. E. Ellis, and D. A. Wolfe. (2012). "Identity development as a buffer of adolescent risk behaviors in the context of peer group pressure and control". Journal of Adolescence 35(4): 917-927. doi:10.1016/j.adolescence.2011.12.012

Foddy, B., and J. Savulescu. (2010). "Relating addiction to disease, disability, autonomy, and the good life." Philosophy Psychiatry \& Psychology 17: 35-42. doi:10.1353/ppp.0.0284

Foster, K. T., B. M. Hicks, W. G. Iacono, and M. McGue. (2015). "Gender differences in the structure of risk for alcohol use disorder in adolescence and young adulthood." Psychological Medicine 45(14): 3047-3058. doi:10.1017/S0033291715001014

Gámez-Guadix, M. (2014). “Depressive symptoms and problematic internet use among adolescents: Analysis of the longitudinal relationships from the cognitivebehavioral model." Cyberpsychology, Behavior, and Social Networking 17 (11): 714-719. doi:10.1089/cyber.2014.0226 
Gámez-Guadix, M., E. Calvete, I. Orue, and C. L. Hayas. (2015). "Problematic Internet use and problematic alcohol use from the cognitive--behavioral model: A longitudinal study among adolescents." Addictive Behaviors 40: 109-114. doi:10.1016/j.addbeh.2014.09.009

Gau, J. M. (2013). "Key characteristics of major depressive disorder occurring in childhood, adolescence, emerging adulthood, and adulthood." Clinical Psychological Science 1(1): 41-53. doi:10.1177/2167702612457599.

Gentile, D. A., H. Choo, A. Liau, T. Sim, D. Li, D. Fung, and A. Khoo. (2011). "Pathological video game use among youths: A two-year longitudinal study." Pediatrics 127(2): e319-329. doi:10.1542/peds.2010-1353

Haagsman, M. C., S. E. Caplan, O. Peters, and M. E. Pieterse. (2013). “A cognitivebehavioral model of problematic online gaming in adolescents aged 12-22 years." Computers in Human Behavior 29: 202-209. doi:10.1016/j.chb.2012.08.006

Haylett, S.A., G. M. Stephenson, and R. M. H. Lefever. (2004) "Covariation in addictive behaviors: A study of addictive orientations using the shorter PROMIS Questionnaire.” Addictive Behaviors 29: 61-71. doi:10.1016/S0306-4603(03)000832

Hoffman, D. L., and T. P. Novak. (2009). "Flow online: lessons learned and future prospects.” Journal of Interactive Marketing 23(1): 23-34. doi:10.1016/j.intmar.2008.10.003

Hox, J. J. (2010). Multilevel analysis: Techniques and applications (2nd ed.). London: Routledge 
Jackson, L. A., A. von Eye, F. Biocca, G. Barbatsis, H. Fitzgerald, and Y.

Zhao. (2003). "Personality, cognitive style, demographic characteristics and Internet use: Findings from the HomeNetToo." Swiss Journal of Psychology 62: 79-90. doi:10.1024//1421-0185.62.2.79

Johansson, A., and K. G. Götestam. (2004). "Internet addiction: Characteristics of a questionnaire and prevalence in Norwegian youth (12-18 years)." Scandinavian Journal of Psychology 45 (3): 223-229. doi:10.1111/j.1467-9450.2004.00398.x

Jones, C. M., L. Scholes, D. Johnson, M. Katsikitis, and M. C. Carras. (2014). “Gaming well: links between videogames and flourishing mental health." Frontiers in Psychology 5: 260. doi:10.3389/fpsyg.2014.00260

Kagitcibasi, C. (2013). “Adolescent autonomy- relatedness and the family in cultural context: What is optimal?" Journal of Research on Adolescence 23(2): 223-235. doi:10.1111/jora.12041

Karacic, S., and S. Oreskovic. (2017). "Internet Addiction Through the Phase of Adolescence: A Questionnaire Study." JMIR Mental Health 4(2): e11. doi:10.2196/mental.5537

Kernan, W. N., C. M. Viscoli, R. W. Makuch, L. M. Brass, and R. I. Horwitz. (1999). "Stratified randomization for clinical trials." Journal of Clinical Epidemiology 52(1): 19-26. doi:10.1016/S0895-4356(98)00138-3

Kim, H. K., and K. E. Davis. (2009). "Toward a comprehensive theory of problematic Internet use: Evaluating the role of self-esteem, anxiety, flow, and the self-rated importance of Internet activities.” Computers in Human Behavior 25 (2): 490-500. doi:10.1016/j.chb.2008.11.001 
Kraut, R., S. Kiesler, B. Boneva, J. N. Cummings, V. Helgeson, and A. M. Crawford. (2002). “Internet paradox revisited.” Journal of Social Issues 58: 49-74. doi:10.1111/1540-4560.00248

Kuss, D., M. D. Griffiths, L. Karila, and J. Billieux. (2014). “Internet addiction: A systematic review of epidemiological research for the last decade." Current Pharmaceutical Design 20(25): 4026-4052.

Kuss, D. J., and M. D. Griffiths. (2012). “Internet gaming addiction: A systematic review of empirical research.” International Journal of Mental Health and Addiction 10(2): 278-296. doi:10.1007/s11469-011-9318-5

Leung, L. (2014). "Predicting Internet risks: a longitudinal panel study of gratificationssought, internet addiction symptoms, and social media use among children and adolescents." Health Psychology and Behavioral Medicine 2(1): 424-439. doi:10.1080/21642850.2014.902316

MacLaren, V. V., and L. A. Best. (2010). "Multiple addictive behaviors in young adults: Student norms for the shorter PROMIS Questionnaire.” Addictive Behaviors 35: 252-255. doi:10.1016/j.addbeh.2009.09.023

Maas, C. J. M., and J. J. Hox. (2004). "Robustness issues in multilevel regression analysis.” Statistica Neerlandica 58(2): 127-137. doi:10.1046/j.0039-0402.2003.00252.x

Maas, C. J. M., and J. J. Hox. (2005). "Sufficient sample sizes for multilevel modeling." Methodology 1(3): 86-92. doi:10.1027/1614-2241.1.3.86 
Micceri, T. (1989). "The unicorn, the normal curve, and other improbable creatures.” Psychological Bulletin 105(1): 156-166. doi:10.1037/00332909.105.1.156

Motti-Stefanidi, F., J. B. Asendorpf, and A. S. Masten. (2012). "The adaptation and wellbeing of adolescent immigrants in Greek schools: A multilevel, longitudinal study of risks and resources." Development and Psychopathology 24: 451-473. doi:10.1017/S0954579412000090

Motto-Stefanidi, F., and A. S. Masten. (2017). “A resilience perspective on immigrant youth adaptation and development." in Handbook on Positive Development of Minority Children and Youth edited by N. Cabrera and B. Leyendecker, 19-34. New York: Springer.

Newman, D. A. (2003). "Longitudinal modeling with randomly and systematically missing data: A simulation of ad hoc, maximum likelihood, and multiple imputation techniques." Organizational Research Methods 6(3): 328-362. doi:10.1177/1094428103254673

Olson, D. H. (2000). “Circumplex model of marital and family sytems.” Journal of Family Therapy 22(2): 144-167. doi:10.1111/1467-6427.00144

Ostovar, S., N. Allahyar, H. Aminpoor, F. Moafian, M. B. M. Nor, and M. D. Griffiths. (2016). "Internet addiction and its psychosocial risks (depression, anxiety, stress and loneliness) among Iranian adolescents and young adults: A structural equation model in a cross-sectional study." International Journal of Mental Health and Addiction 14(3): 257-267. doi:10.1007/s11469-015-9628-0 
Poli, R., and E. Agrimi. (2012). "Internet addiction disorder: Prevalence in an Italian student population.” Nordic Journal of Psychiatry 66(1): 55-59. doi:10.3109/08039488.2011.605169

Raudenbush, S. W., and A. S. Bryk. (2002). Hierarchical linear models: Applications and data analysis methods (2nd ed.). Thousand Oaks, CA: Sage Publications, Inc.

Rettie, R. (2001). “An exploration of flow during Internet use.” Internet Research 11(2): 103-113. doi:10.1108/10662240110695070

Rohde, P., P. M. Lewinsohn, D. N. Klein, J. R. Seeley, and J. M. Gau. (2013). “Key characteristics of major depressive disorder occurring in childhood, adolescence, emerging adulthood, and adulthood." Clinical Psychological Science 1(1): 41-53. doi:10.1177/2167702612457599.

Rosenberg, M. (1965). Society and the adolescent self-image. Princeton, N.J.: Princeton University Press.

Schwarzer, R. (1993). Measurement of perceived self-efficacy: Psychometric scales for cross-cultural research. Berlin, Germany: Freie Universität Berlin.

Shaw, M., and D. W. Black. (2008). “Interned addiction.” CNS Drugs 22: 353. doi:10.2165/00023210-200822050-00001

Shi, X., J. Wang, and H. Zou. (2017). "Family functioning and Internet addiction among Chinese adolescents: The mediating roles of self-esteem and loneliness." Computers in Human Behavior 76: 201-210. doi:10.1016/j.chb.2017.07.028

Shulman, E. P., K. P. Harden, J. M. Chein, and L. Steinberg. (2015). “Sex differences in the developmental trajectories of impulse control and sensation-seeking from early 
adolescence to early adulthood." Journal of Youth and Adolescence 44(1): 1-17. doi:10.1007/s10964-014-0116-9

Society of Information Observatory. (2010). “Online Statistics eEurope 2005/i2010.” Accessed November, 2015. http://stats.observatory.gr/pxweb2006/Database /eEurope/1_noikokyria/3_ekpaideysi_fylo_ilikia/3_ekpaideysi_fylo_ilikia.asp

Stavropoulos, V., K. Alexandraki, and F. Motti-Stefanidi. (2013a). "Recognizing internet addiction: Prevalence and relationship to academic achievement in adolescents enrolled in urban and rural Greek high schools." Journal of Adolescence 36(3): 565576. doi:10.1016/j.adolescence.2013.03.008

Stavropoulos, V., K. Alexandraki, and F. Motti-Stefanidi. (2013b). "Flow and telepresence contributing to Internet abuse: Differences according to gender and age." Computers in Human Behavior 29(5): 1941-1948. doi:10.1016/j.chb.2013.03.011

Stavropoulos, V., D. J. Kuss, M. Griffiths, and F. Motti-Stefanidi. (2016a). “A longitudinal study of adolescent internet addiction: the role of conscientiousness and classroom hostility." Journal of Adolescent Research 31(4): 442-473. doi:0743558415580163

Stavropoulos, V., D. Gentile, and F. Motti-Stefanidi. (2016b). “A multilevel longitudinal study of adolescent Internet addiction: The role of obsessive-compulsive symptoms and classroom openness to experience.” European Journal of Developmental Psychology 13(1): 99-114. doi:10.1080/17405629.2015.1066670

Stavropoulos, V., D. J. Kuss, M. D. Griffiths, P. Wilson, and F. Motti-Stefanidi. (2017a). "MMORPG gaming and hostility predict Internet Addiction symptoms in adolescents: An empirical multilevel longitudinal study." Addictive Behaviors 64: 294-300. doi:10.1016/j.addbeh.2015.09.001 
Stavropoulos, V., Gomez, R., Steen, E., Beard, C., Liew, L., \& Griffiths, M. D. (2017b).

The longitudinal association between anxiety and Internet addiction in adolescence: The moderating effect of classroom extraversion. Journal of Behavioral Addictions, 1-11. https://doi.org/10.1556/2006.6.2017.026

Steinberg, L. (2007). “A social neuroscience perspective on adolescent risk-taking.” Developmental Review 28: 78-106. doi:10.1016/j.dr.2007.08.002

Sui, D., M. Goodchild, and S. Elwood. (2013). "Volunteered geographic information, the exaflood, and the growing digital divide: Volunteered geographic information (VGI) in theory and practice." In Crowdsourcing geographic knowledge, edited by D. Sui, S. Elwood, and M. Goodchild, 1-12. Netherlands: Springer. doi:10.1007/978-94007-4587-2_1

Sussman, S., and A. N. Sussman. (2011). "Considering the definition of addiction." International Journal of Environmental Research and Public Health 8(10): 40254038. doi:10.3390/ijerph8104025

Tsagarakis, M., K. Kafetsios, and A. Stalikas. (2007). "Reliability and validity of the Greek version of the Revised Experiences in Close Relationships measure of adult attachment." European Journal of Psychological Assessment 23(1): 47-55. doi:10.1027/1015-5759.23.1.47

Van Deursen, A. J., and J. A. Van Dijk. (2014). “The digital divide shifts to differences in usage." New Media and Society 16(3): 507-526. doi:10.1177/1461444813487959

Williams, D (2015). "The health of men: Structured inequalities and opportunities." American Journal of Public Health 93(5): 724-731. doi:10.2105/AJPH.93.5.724 
Willoughby, T. (2008). “A short-term longitudinal study of Internet and computer game use by adolescent boys and girls: Prevalence, frequency of use, and psychosocial predictors.” Developmental Psychology 44(1): 195-204. doi:10.1037/00121649.44.1.195

Witmer, B. G., and M. J. Singer. (1998).” Measuring presence in virtual environments: Presence questionnaire". Presence, Teleoperators and Virtual Environments 7: 225240. doi:10.1162/105474698565686

Yang, S., Y. Lu, B. Wang, and L. Zhao. (2014). "The benefits and dangers of flow experience in high school students' internet usage: The role of parental support." Computers in Human Behavior 41: 504-513. doi:10.1016/j.chb.2014.09.039

Yen, C-F., C-H. Ko, J-Y. Yen, Y-P. Chang, and C-P. Cheng. (2009). "Multi-dimensional discriminative factors for Internet addiction among adolescents regarding gender and age." Psychiatry and Clinical Neurosciences 63(3): 357-364. doi:10.1111/j.1440-1819.2009.01969.

Young, K.S. (1998). Caught in the Net. New York: Wiley

Yu, L., and D. T. L. Shek. (2013). "Internet addiction in Hong Kong adolescents: A threeyear longitudinal study.” Journal of Pediatric and Adolescent Gynecology 26 (3): S10-S17. doi:10.1016/j.jpag.2013.03.010

Zaman, M., A. Murugan, and D. Qizhi. (2010). "Experiencing flow with instant messaging and its facilitating role on creative behaviors." Computers in Human Behavior 26: 1009-1018. doi:10.1016/j.chb.2010.03.001 
Note 1: The present data have been used in three more published studies that address different theoretical questions (Stavropoulos, Kuss, Griffiths \& Motti-Stefanidi, 2016b; Stavropoulos, Gentile \& Motti-Stefanidi 2016b; Stavropoulos, Kuss, Griffiths, Wilson \& Motti-Stefanidi, 2017). Instruments used in the data include the: (i) Internet Addiction Test IAT (Young, 1998); (ii) Presence II questionnaire (Witmer \& Singer, 1998); (iii) Online Flow Questionnaire (Chen, Wigand \& Nilan, 1999); (iv) Symptom Check List 90 (Derogatis \& Savitz, 1994); (v) Rosenberg Self-Esteem Scale (Rosenberg, 1965); (vi) Five Factor Questionnaire for Children (FünfFaktoren-Fragebogen für Kinder) (Asendorpf \& Van Aken, 2003); (vii) Generalized Self-Efficacy Scale (Schwarzer, 1993); (viii) Family Adherence and Cohesion Evaluation Scale (Olson, 2000); (ix) Socio-metric Questionnaire (Coie, Dodge \& Coppotelli, 1982); (x) Greek version of the Experience of Close Relationships Revised (Tsagarakis, M., Kafetsios, K., \& Stalikas, A., 2007); (xi) demographic and internet use questions and; (xii) school grades of the participants were retrieved from their school records. 
Note 2: The data abides with the sample size requirements suggesting: a) a minimum ratio of $10_{\text {clusters }} / 5_{\text {participants }}$ to test for fixed effects and cross-level interactions in models with one explanatory variable at each of the levels, and: b) a minimum requirement of 30 clusters for testing standard errors of fixed effects (Maas \& Hox, 2004, 2005).

Note 3: Conducting covariance based structural equation modeling (CBSEM) was not selected as: a) it requires at least three or four indicators (the current study includes two time points) for every latent variable (growth) (Baumgartner \& Homburg, 1996) and; b) it assumes multi-normal distribution of the observed variables to ensure meaningful results-which is rarely the case in empirical research (Micceri, 1989). Similarly, latent growth modeling (LGM) was not chosen as it assumes that Level 1 predictors with random effects have the same distribution across all participants in each subpopulation-while HLM allows different distributions (Raudenbush \& Bryk, 2002). Finally, HLM was preferred over partial least square analysis (PLS), as it estimates the effects of variables on the outcome variable at one level (i.e. individual), while at the same time taking into account the effect of variables on the outcome variable at another level (i.e. classroom) (Raudenbush \& Bryk, 2002). 